\title{
The AutoDrive Challenge: Autonomous Vehicles Education and Training Is- sues
}

\section{Dr. Jennifer Melanie Bastiaan, Kettering University}

Jennifer Bastiaan received her Ph.D. in Mechanical and Mechatronics Engineering from the University of Waterloo. She is an Assistant Professor in the Mechanical Engineering department at Kettering University, where she is focused on teaching and research in ground vehicle systems. She is a veteran of the U.S. automotive industry with two decades of experience, including modeling and physical testing programs. Her technical research interests include vehicle dynamics, tire mechanics, and sound and vibration. She is also interested in education research, especially regarding the design of engaging STEM outreach programs for pre-college students, and the incorporation of effective industry partnerships into undergraduate engineering education.

\section{Dr. Diane L. Peters, Kettering University}

Dr. Peters is an Assistant Professor of Mechanical Engineering at Kettering University. She is the primary faculty advisor for the university's AutoDrive team.

\section{Dr. Juan R. Pimentel, Kettering University}

Dr. Juan R. Pimentel is a Professor of Computer Engineering at Kettering University in Flint, Michigan, USA. He is an expert in the area of Internet of Things, Industrial Internet, safety-critical systems and industrial computer networks, particularly issues related to real-time protocols, safety-critical protocols, dependable automotive embedded distributed systems, and distributed industrial and embedded systems. $\mathrm{He}$ is a recognized international expert in the area of industrial communications and real-time and dependable protocols. He has written 3 books on networking, multimedia systems, and safety-critical systems. He has worked with major manufacturing and process control projects involving products from companies such as Siemens, Rockwell, Schneider Electric, ABB, and GE-Fanuc. He has also prepared and delivered seminars in Europe, Middle East, North and South America in areas such as Distributed Control Systems, SCADA, Communication networks, wireless industrial networks and protocols, Process monitoring and control, process safety, sensors and instrumentation, and information management. As a 1980 graduate of the University of Virginia, his accomplishments include the co-development of the application layer for Profibus (with Siemens), and the development of FlexCAN, a CAN-based safety-critical architecture. He has performed research at well known institutions around the world such as the Franuhofer Institute at Karlsruhe-Germany, INRIA at Nancy-France, University of Padova in Italy, Universidad Polictecnica de Madrid in Spain, Universidad Carlos III de Madrid in Spain, Universidad de los Andes, Bogota-Colombia, and UTEC, Lima, Peru. In 2007 he received the "Distinguished Researcher Award" from Kettering University for contributions in the area of industrial communication systems and automotive systems. During the last few years he has been involved with wireless sensor networks (WSNs), telemetry systems using tv white spaces, software define radios (SDR), and platforms for deploying IoT technologies.

\section{Dr. Mehrdad Zadeh, Kettering University}

Dr. Zadeh is an associate professor and an advisor of AutoDrive Challenge ${ }^{\mathrm{TM}}$ competition at Kettering University, MI. From Sept. 2015 to January 2017, he served as a visiting associate professor at Johns Hopkins University, Laboratory for Computational Sensing + Robotics (LCSR), MD, where he collaborates on the design and development of haptic-enabled surgical simulation. He received his M.A.Sc. (2004) and Ph.D (2009) in Electrical and Computer Engineering from Concordia University and the University of Waterloo, Canada, respectively. He also has over 8 years of industry experience as an engineer and software development specialist. He is interested largely in the areas of autonomous systems, haptics, physical human robot interaction (pHRI), and Robotics. 


\title{
The AutoDrive Challenge at Kettering University: Autonomous Vehicles Education and Training Issues
}

\begin{abstract}
Automotive companies are focusing significant research and development efforts on autonomous vehicles. As they do so, they recognize the need for a large, well-trained workforce that is equipped to conduct these research and development projects, particularly in light of the projected shortages of STEM professionals in the United States. Some of these companies have found various ways to engage with professional societies and with universities to encourage the development of this workforce, and to promote themselves to STEM students while they are still in school. One such effort is the SAE / GM AutoDrive Challenge ${ }^{\mathrm{TM}}$, a new collegiate competition organized by SAE International in collaboration with General Motors Corporation. In this competition, eight teams are working to modify a Chevrolet Bolt to meet the requirements of a Level 4 autonomous vehicle (i.e., a vehicle that is totally capable of driving itself within a certain operational domain). Teams were selected for this competition through a proposal process, with one of the requested components of the proposal focusing on existing courses and the development of new courses at the participating university. In this paper, we will discuss the roles of students and faculty advisors at one of the participating schools, address issues related to education and training of students who want to work in the autonomous vehicle industry, and discuss the benefits of the competition to all of its stakeholders. This discussion will include the skills developed by students, the outcomes of the competition, and the value that is being created for the automotive industry. As part of this discussion, we will focus on the close ties that can be forged between the participating universities and the corporate sponsors of the AutoDrive Challenge, as well as the impact on course development at the university.
\end{abstract}

\section{Introduction}

Autonomous, self-driving vehicles are currently receiving a lot of attention from manufacturers, suppliers, service providers, governments, universities, start-ups, and other stakeholders. There have been several autonomous vehicle competitions in the past that have sparked much interest and have contributed to several advances in the technologies behind self-driving vehicles [1,2]. One of the latest competitions is the AutoDrive Challenge ("AutoDrive"), managed by a partnership between SAE International ("SAE") and General Motors Corporation ("GM"). This paper briefly describes AutoDrive, the role of students and faculty advisors at Kettering University, the main outcomes of the competition, and issues involving the education and training in the area of autonomous driving. All authors are faculty advisors to the Bulldog Bolt, the name of the group from Kettering University participating in AutoDrive. Kettering University is one of eight institutions selected to participate in the three-year long competition that began in April 2017.

Accordingly, the main goals of the paper are to summarize AutoDrive, explain the role played by student and faculty advisors, identify the main outcomes of the competition, and address issues involving education and training for the design of autonomous vehicles. This will involve answering a number of important questions such as: What skills will be learned by the students? What is the value of the competition? What is the state of education and training for autonomous 
vehicles design around the world? What are the important topics for educating and training future self-driving engineers? What are the main challenges in designing and offering education and training programs for preparing self-driving engineers?

Student competitions have been shown to have significant educational benefits [3-6], such as allowing students to put their coursework into practice [7], particularly when this practice can be integrated into the engineering curriculum, as has been done at times with Formula SAE [8]. Some of the skills the competitions promote are an entrepreneurial mindset, project management, leadership skills [9], and systems engineering knowledge [10]. AutoDrive is not only one of the latest collegiate competitions, but perhaps it is the most challenging competition involving autonomous vehicles at a high level of complexity, defined as SAE Level 4 autonomy, per SAE Standard J3016 [11]. One of the related issues to address is the education and training of students directly participating in the competition and more generally, of students interested in a future career in the area of autonomous vehicles.

Many approaches exist to design and deliver education and training programs that can be suitable for autonomous driving. For example, there has been a considerable amount of research on problem-based learning, including the use of mobile robots and autonomous vehicles in class projects. These types of projects draw on interest in real-life applications, which has been shown to enhance engineering education $[12,13]$. These projects have covered the pre-college age range, including students in elementary school [14] and middle school [15], as well as students in undergraduate engineering programs. In one case, a wireless autonomous vehicle was used in a collaboration involving four-year universities, two-year colleges, and pre-college students [16]. In undergraduate engineering programs, autonomous vehicles have been used for freshman classes [17] all the way through senior design (capstone) projects $[18,19]$. The autonomous vehicles have a variety of different scales and designs, including solar-powered vehicles [20], wireless vehicles, and a vehicle with a focus on infrared sensing [21]. Because of space considerations, we do not provide a summary or review of the technologies underlying autonomous vehicles, as they are available in a number of publications on the subject [22-25].

\section{AutoDrive at Kettering University}

Participation in SAE programs is a large part of the education of many students at Kettering University. The institution has traditionally supported four SAE Collegiate Teams ("Teams"), including SAE Aero Design, Baja SAE, Clean Snowmobile Challenge and Formula SAE. Starting in 2017, a fifth Team, dedicated to AutoDrive, has been officially added to the Teams supported by Kettering University. All Teams are associated with the official SAE Collegiate Design Series ("Series"). AutoDrive is the newest Series for SAE. SAE and GM are co-sponsoring this three-year competition. The competition challenges students to develop a fully autonomous passenger car, based on an existing production electric vehicle, the 2017 Chevrolet Bolt. The objective of the competition is to have the vehicle successfully travel through a city driving course autonomously. The level of autonomy required by the end of the three-year competition cycle is SAE Level 4.

The AutoDrive Challenge differs from other Series in that only eight Teams are participating. The eight teams were selected via a pre-competition in which educational institutions were invited to submit a proposal. The eight Teams approved for AutoDrive are: Kettering University, Michigan 
State University, Michigan Technological University, North Carolina A \& T State University, Texas A \& M University - College Station, University of Toronto, University of Waterloo, and Virginia Polytechnic Institute. Figure 1 shows photographs of all the AutoDrive Teams and the Kettering University Team. The Kettering University Team consists of eleven faculty advisors and approximately sixty undergraduate and graduate students; not all of these participants are shown in the picture.

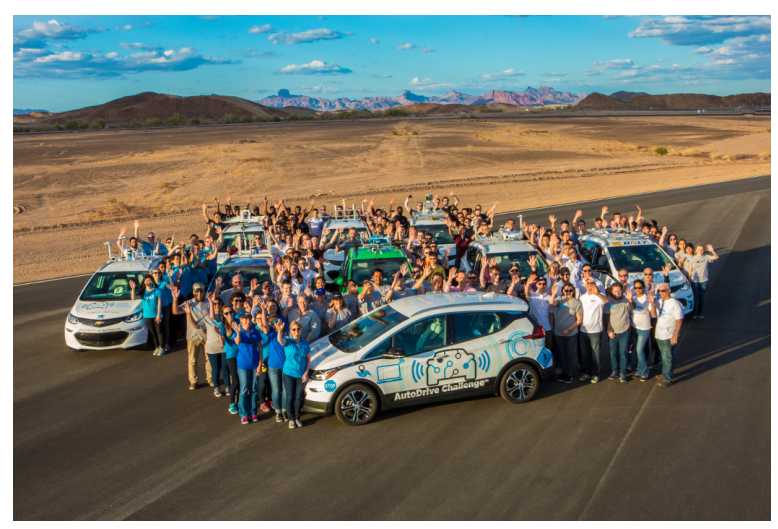

(a) All Teams

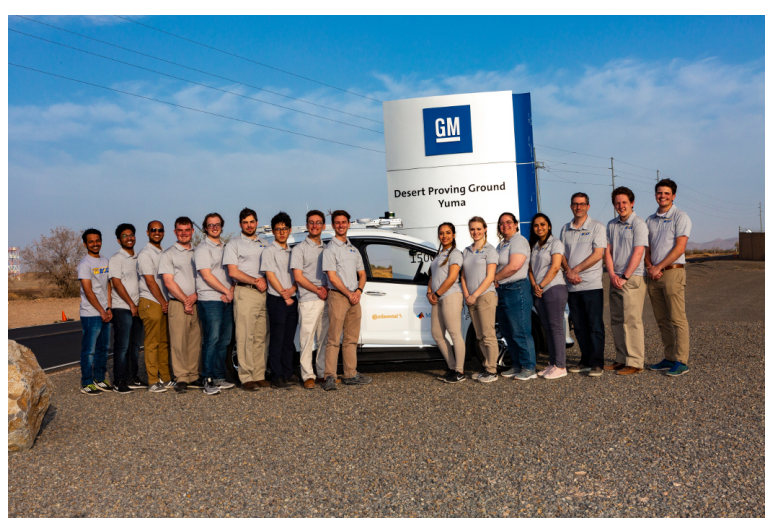

(b) Kettering University Team

Figure 1: Year 1 AutoDrive Teams

AutoDrive is providing students with practical engineering experience in replacing an organic human driver with an inorganic autonomous driver. The Chevrolet Bolt vehicle is being modified from its production form, in which manual control by a human being is required, to its final competition form in which the vehicle is under full automatic control of the autonomous driver, as defined by SAE Level 4 . The many application areas to be addressed include, but are not limited to, sensor installation, data acquisition, signal processing, filter design, artificial intelligence, image processing, control design, computer software programming, and computer hardware integration. Other, more general, concerns must also be addressed in the vehicle design, such as the noise and vibration performance of the autonomous vehicle, and its cosmetic appearance.

In order to help with the design and fabrication of the autonomous vehicle build, a number of co-sponsors have donated software and equipment to the Kettering University AutoDrive Team. Major in-kind equipment donations include an Intel computing platform, Velodyne LIDAR devices and a Continental RADAR system. It is the responsibility of Kettering University students to make good use of these donations, as faculty advisors are strictly prohibited from directly participating in the design of the autonomous vehicle. AutoDrive is a student competition; faculty advisors may provide general engineering advice, but they may not design, manufacture, or repair any part of the vehicle. This restriction also excludes faculty advisors from creating documentation or modifying the vehicle's on-board computer system. Each yearly competition as part of AutoDrive will become increasingly difficult. Details of the first year competition are described below.

\section{II.A First Year Competition (April 2018)}

The first year competition consisted of creating a series of written documents, participating in oral presentations, and competing in a sequence of dynamic events at the competition venue in Yuma, 
Arizona, U.S.A. The total number of points possible in the first year was 1000. Each of the three competition years have a total of 1000 possible points. At the end of the three year competition cycle, the team that has accumulated the greatest number of points with respect to the possible 3000 points will be declared the overall winner of AutoDrive. The 1000 points available during the first year were divided into "static events" (400 points), "technical reports" (150 points), and "dynamic events" (450 points).

The purpose of the static events and technical reports were to provide the Teams with an early opportunity to communicate their design process and engineering plans to event organizers in advance of the on-site competition in Yuma. All of the static events and technical reports had deadlines in advance of the April 2018 competition, during which the dynamic events were performed. The content of the static events and technical reports were supposed to include a description of how each Team intended to implement the automated driving system in the vehicle, with sufficient detail to satisfy the organizers and judges that the students were making a serious engineering effort to design the autonomous vehicle. Safety and social concerns related to autonomous driving were also to be addressed, including a discussion of the ethical and moral dilemmas faced by an autonomous driver and how these decisions should be made. Any Team not demonstrating good engineering practices or submitting a frivolous report could be disqualified by the organizers at any time in the competition.

Teams that demonstrated satisfactory performance in the static events and technical reports were permitted to participate in the dynamic events in Yuma. A description of the dynamic events follows. All of the on-road dynamic events were supposed to performed with the vehicle in autonomous mode. Each vehicle operating in autonomous mode required a student safety driver who rode on-board in the driver seat and who was ready to resume manual control of the vehicle in the event of a failure of the autonomous driving system. The autonomous mode of operation was indicated by two blue lights, one on the exterior of the car on the roof, and one in the interior of the car. When the blue lights were on, this indicated to observers, whether inside or outside the vehicle, that the autonomous driver was controlling the vehicle. Figure 2 shows the Kettering University Team in action at the Yuma competition.

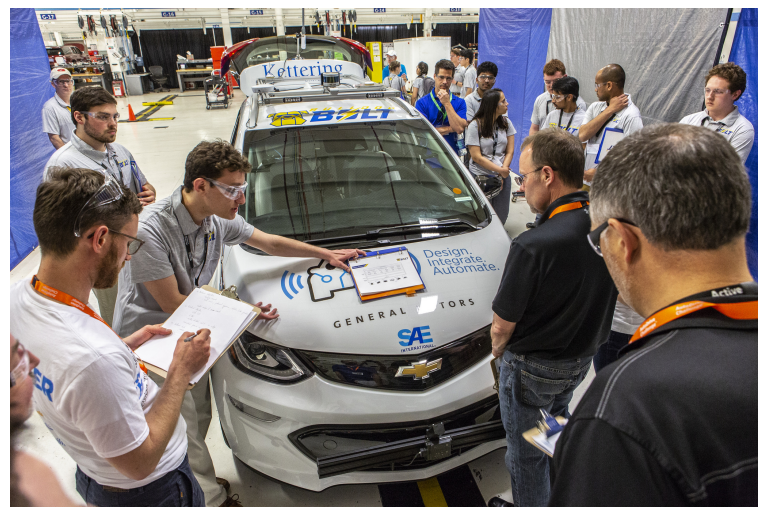

(a) Oral Examination by Volunteer Judges

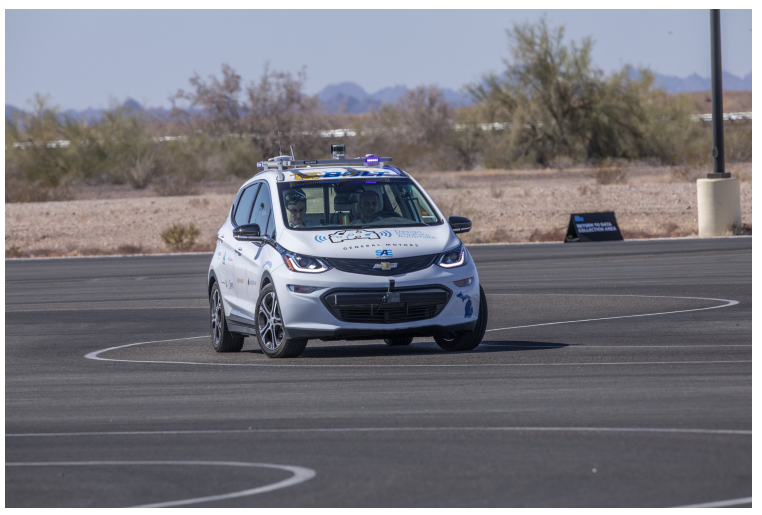

(b) On-Road in Autonomous Mode

Figure 2: Kettering University AutoDrive Team at Year 1 Competition 


\section{Mapping Challenge}

In the first year competition, the mapping challenge was conducted using laptop computers, rather than in the vehicle. Teams were provided with a map database for North America. Teams were tasked with creating software that searches and displays the contents of the map database. During the mapping challenge event, 25 separate searches were conducted by the Teams. Teams were provided with the searches at the competition, which included street addresses and attractions. The mapping system designed by the Team had to pinpoint the desired locations within one block. The system was also supposed to be able to "zoom out" to higher points of view, ultimately including neighboring streets and highway systems. In the second and third years of the competition, the mapping software designed by the Teams will be integrated into the vehicle and employed as part of the autonomous driving system.

\section{Longitudinal Stop Line Challenge}

This was a straight-line only event representing typical city driving on a straight road. The course consisted of a straight road of about 0.5 mile in length with 3 stop signs along the route. The single lane of travel was 11.5 feet wide. The road demarcation lines indicating the sides of the lane were $4-6$ inch in width, and the lines could have been solid, dashed, or any combination of the two (Teams were not given this information in advance of the competition). The autonomous vehicle was required to identify and process information related to road demarcation lines and stop signs, with the goal of driving the route in a manner consistent with the legal limits of the competition. Specific rules were provided that governed the acceptable stopping distances from stop lines, the acceleration and deceleration limits of the vehicle, the jerk limits of the vehicle and the maximum travel speed of the vehicle. Teams were scored based on their compliance with these legal limits. In general, the limits of this and the other dynamic tests were designed to represent the behavior of a well experienced driver, where smooth operation of the vehicle is preferred rather than herky-jerky motions. Thus, the ideal autonomous driver, according to the rules of the competition, has more in common with a middle-aged chaffeur than a teenager taking their first driving lessons.

\section{Lateral Challenge}

This was a low-speed cornering event with two separate courses. The first course had a square-wave appearance (in plan view), with very tight turns. Lane width could have been as low as 10 feet; lane markings were $4-6$ inch wide. There was a total of six complete cycles of the same square wave road geometry pattern that had to be negotiated autonomously by the vehicle in order to obtain maximum points. If all four wheels traveled outside of the lane, the Team was disqualified, which was true for all of the dynamic events. The second course had turns of varying radii; thus, it did not present a consistent, repeatable route as the first course did. Similar legal limits applied to the lateral challenge as applied to the longitudinal challenge, where there were restrictions on speed, acceleration and jerk. Teams were scored based on how closely the autonomous vehicle conformed to the published limits.

\section{Object Detection \& Avoidance Challenge}

Teams had to successfully complete the lateral challenge in order to qualify for the object avoidance challenge. The object avoidance challenge consisted of a straight course with three adjacent lanes. Static objects were placed around the course in random locations that were unknown to the teams in advance of the competition. Some objects blocked an entire travel lane; 
other objects were located at the side of the road. The objective was to have the autonomous vehicle successfully travel through the course without colliding with any of the objects. If the travel lane of the autonomous vehicle was blocked by an object, it was expected that the autonomous vehicle would change lanes and go around the obstacle, assuming there was an open, adjacent travel lane. In addition to obstacles located at the road-side, there were also be standard road signs located there, such as speed limit signs. Points were awarded for correct identification and classification of road signs.

\section{Year 1 Results}

Table 1 lists the overall results for the first year of the AutoDrive Challenge for all of the Teams. The total points earned by each team, out of 1000 possible points, is listed. The Kettering University AutoDrive Team had a very respectable fourth place finish.

Table 1: AutoDrive Challenge Year 1 Results

\begin{tabular}{ccc}
\hline Place & School & Total Points \\
\hline \hline 1 & University of Toronto & 792 \\
\hline 2 & Texas A \& M University & 690 \\
\hline 3 & Virginia Polytechnic Institute & 673 \\
\hline 4 & Kettering University & 523 \\
\hline 5 & University of Waterloo & 515 \\
\hline 6 & North Carolina A \& T State University & 475 \\
\hline 7 & Michigan State University & 447 \\
\hline 8 & Michigan Technological University & 445 \\
\hline
\end{tabular}

\section{Student Education and Training Needs}

In the case of AutoDrive, at Kettering University the relationship between student coursework and the competition was explicitly considered from the very start, as the university's proposal was developed. In the evaluation of existing courses, there are several existing Kettering University courses that are relevant to AutoDrive. These include fundamental courses in dynamic systems, basic electrical and computer engineering topics, and computer science courses. In addition to those foundation courses, many of them required within the relevant major, there are classes with special relevance to automotive engineering and to autonomous vehicles.

In deciding which courses were relevant, we considered autonomous vehicles as a broad, interdisciplinary area, requiring good design of the physical system and its dynamic characteristics, a well-chosen control scheme, appropriate sensors, and effective programming of the actual system. If any one of those elements is missing or not properly integrated with the others, the system will suffer. Therefore, courses from a variety of disciplines were selected as being pertinent. Some of the more specialized existing classes included Automotive Control Systems, Vehicle System Dynamics, Digital Control Systems, Robot Dynamics and Control, Advanced Digital Signal Processing, Introduction to Mobile Robotics, Embedded Systems, and Artificial Intelligence. 
Additional courses were also proposed, in order to provide students with additional expertise that is needed for AutoDrive; these include a one-credit introductory course for the competition and classes in Sensors and Actuators and Computer Vision. A continuing assessment was also proposed, in order to determine what classes could be developed. Many other topics, such as mission planning, path planning, and diagnostics could be integrated into existing courses.

Part of the process of recruiting and training undergraduate student team members involved the one-credit course mentioned above, which was graded on a credit / no credit basis. This course, Introduction to AutoDrive, was taught as a special topics class. In order to enroll in the class, students had to apply to be on the AutoDrive Team. The Team application required them to specify areas of interest in the competition as well as provide a resume. The class met for six weeks, which is just over half of the school term; with the class time allocated to the early part of the term, students were able to start working effectively on the team as early as possible. The course includes information that students need in order to come up to speed - an introduction to the rules and progress to date on the project, safety procedures when working on the project, rights and obligations under the various Non-Disclosure Agreements ("NDA"s) that the students need to sign, file and data management on the project, choosing and learning about a sub-team, and social responsibility aspects of autonomous vehicles. At the end of the class, students were asked to fill out a voluntary survey. Per the Institutional Review Board ("IRB") approval, the instructor for the course was not aware of who did or did not fill out the survey. Completed surveys were turned in to an administrative person, who removed names and other identifying information and retained the informed consent forms.

As part of the survey, students were asked about their interest in taking an Independent Study course related to AutoDrive, and also in courses relevant to AutoDrive that they might wish to see offered in the future. The education and training needs of students, therefore, are being evaluated from two perspectives: that of the students, who can speak to their interests, and that of the faculty, who are acquainted with the state of the art in the field. Student comments indicated that they are interested in additional courses (or material) on safety, vehicle dynamics and control, vehicle operations, sensors, programming and coding for autonomous vehicles, and automotive design.

It is noted that many of the classes mentioned by students are courses that either exist or are on the list of classes that faculty are interested in developing. Another notable feature in student comments was a concern over pre-requisite chains, as students recognize the need to balance disciplinary knowledge and the issues of scheduling courses for the broadest possible appeal. Of course, students at an early stage of the competition may not realize what other topics may be useful and interesting, such as sensor fusion, machine vision, and neural networks. Therefore, an additional, follow up survey was conducted during the second year of competition; this survey was distributed to all team members, both undergraduate and graduate students. Per IRB approval, the survey was anonymous, and the only information collected about the students was their major and class standing. Twenty-one valid responses were received.

In addition to information about their class standing and major, the survey asked students questions about their level of involvement, new classes they would be interested in taking, what material they would like to see integrated into existing classes, their interest level in independent study, thesis options, and AutoDrive related capstone classes. Students were also asked about 
their interest in and preparation for working in the field of autonomous vehicles. The students who responded represented several different majors and all class standings, although the majority of respondents were seniors. The students' majors are listed in Table 2, with their class standing shown in Figure 3. Out of the 21 students who provided valid responses, five of them are dual major students.

Table 2: Majors of Respondents

\begin{tabular}{cc}
\hline Major & Percentage $^{\mathrm{a}}$ \\
\hline Computer Engineering & $33 \%$ \\
\hline Computer Science & $24 \%$ \\
\hline Electrical Engineering & $38 \%$ \\
\hline Mechanical Engineering & $29 \%$ \\
\hline${ }^{a}$ Total is greater than $100 \%$ due to dual majors.
\end{tabular}

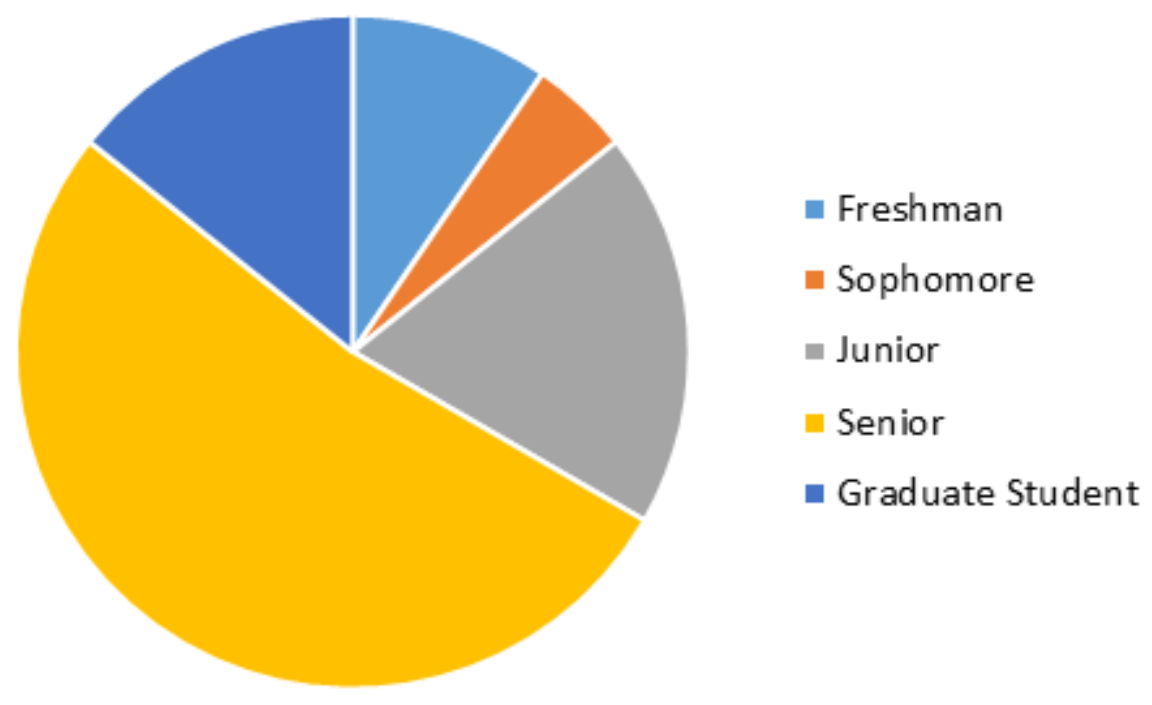

Figure 3: Class Standing of Respondents

The level of involvement of the respondents also varied; students were asked to self-identify their level of involvement on a five-point Likert scale, where 1 represented the lowest level of involvement and 5 was the highest level of involvement. The average was 3.29, with the distribution shown in Figure 4.

Students were asked the question, "Assuming that it would fit in your schedule, how interested would you be in taking classes specifically on the following topics?" They were presented with a list of 11 different options that they could rate on a scale of 1 to 4 , with 1 representing "not at all interested" and 4 denoting "very interested". Every topic was of strong interest to at least some students, and all topics had an average interest level greater than 2. The ranked list of topics, with the average rating given, is in Table 3. 


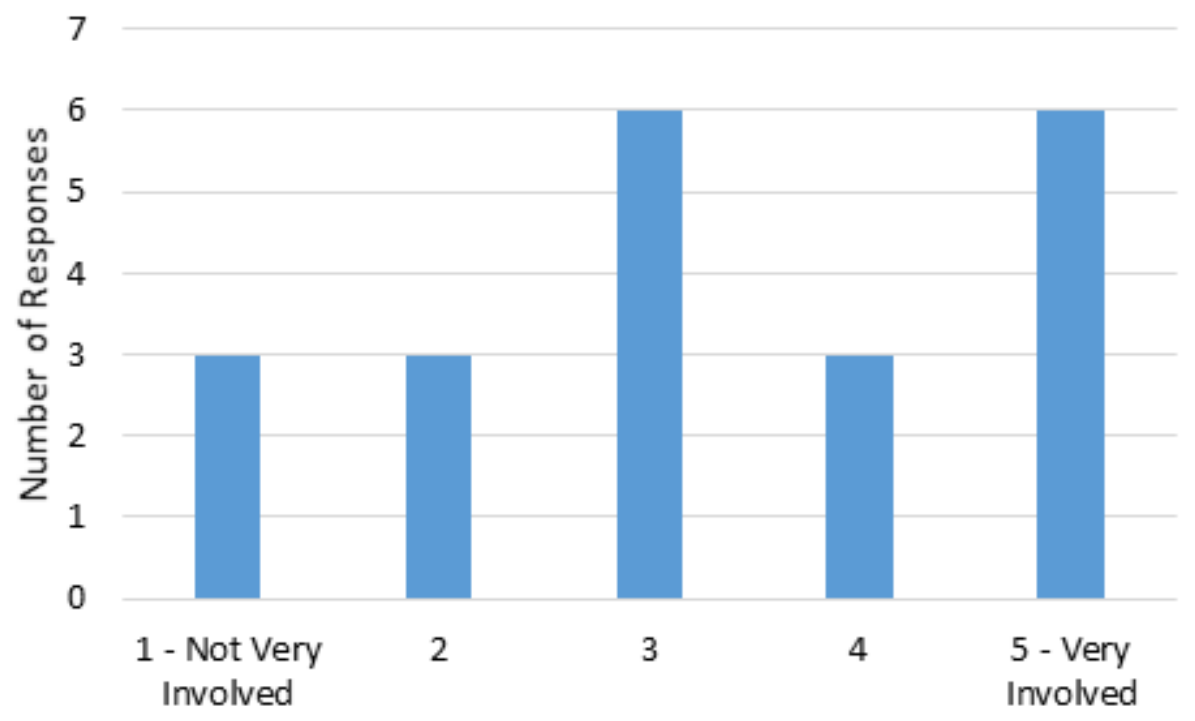

Figure 4: Level of Involvement

The topics of strongest interest were control of autonomous vehicles and artificial intelligence, respectively. Control of autonomous vehicles was of broader interest, with no significant difference between the average interest across majors. Artificial intelligence, in contrast, was of stronger interest to certain majors. Computer engineers had a very strong interest in it, with an average rating of 3.86, and mechanical engineers less interested, with a rating of 2.67. This suggests that certain courses should be designed as interdisciplinary courses, while others may be more discipline-specific, although students in other majors may wish to take them as technical electives.

Students were also asked the question, "How interested are you in seeing the following topics integrated into existing classes that you will be taking?" They were presented with the same list of options. The averages tended to be higher, indicating that students have a slightly stronger interest in seeing their existing courses enhanced with autonomous related topics. The top choices were the same as for the creation of new courses, with small changes in the ranking occurring in the lower ranked items. However, the distinction between items is small in many cases, and therefore these differences are not significant, given the number of survey responses. The ranked list of integration topics, with the average rating given, is in Table 4.

Students were then asked to rate their level of interest, again on a four-point scale, in three options: completing an independent study on AutoDrive, taking a capstone class on AutoDrive, and completing an undergraduate or graduate thesis on AutoDrive. The two options of completing an independent study and taking a capstone class were the more popular options, with average ratings of 3.06 and 3.05, respectively. Completing a thesis on AutoDrive had an average rating of 2.80 , although it was far more popular with graduate students, with all the graduate students saying they were very interested in this option. Taking a capstone class was of less interest to graduate students, with an average rating of 2.33. This is not surprising, given the relative importance and applicability of a capstone class to undergraduate versus graduate students. Both 
Table 3: Interest in New Courses

\begin{tabular}{lll}
\hline Rank & Topic & Rating \\
\hline 1 & $\begin{array}{l}\text { Control of autonomous vehicles (including predictive control and other modern } \\
\text { control techniques) }\end{array}$ & 3.29 \\
\hline 2 & Artificial intelligence (neural networks, fuzzy logic, soft computing approaches) & 3.24 \\
\hline 3 & Sensors / sensor fusion (camera, LIDAR, RADAR, IMU) & 3.14 \\
\hline 4 & Computer vision & 3.00 \\
\hline 5 & Vehicle dynamics (mathematical modeling of vehicle behavior) & 2.67 \\
\hline 5 & Dynamic systems modeling in MATLAB / Simulink & 2.67 \\
\hline 7 & Use of the Autonomous Vehicle toolbox in MATLAB / Simulink & 2.57 \\
\hline 8 & Vehicle on-board communication systems (Ethernet, CAN, CAN-FD, USB) & 2.43 \\
\hline 9 & Project and personnel management & 2.33 \\
\hline 10 & Self-driving vehicle ethics and legal issues & 2.29 \\
\hline 11 & Functional safety & 2.24 \\
\hline 12 & Other & 2.12 \\
\hline
\end{tabular}

Table 4: Interest in Integration of Autonomous Related Content Into Existing Courses

\begin{tabular}{lll}
\hline Rank & Topic & Rating \\
\hline 1 & $\begin{array}{l}\text { Control of autonomous vehicles (including predictive control and other modern } \\
\text { control techniques) }\end{array}$ & 3.52 \\
\hline 2 & Artificial intelligence (neural networks, fuzzy logic, soft computing approaches) & 3.29 \\
\hline 3 & Sensors/sensor fusion (camera, LIDAR, RADAR, IMU) & 3.14 \\
\hline 4 & Vehicle dynamics (mathematical modeling of vehicle behavior) & 3.10 \\
\hline 5 & Dynamic systems modeling in MATLAB / Simulink & 3.05 \\
\hline 6 & Computer vision & 2.95 \\
\hline 6 & Vehicle on-board communication systems (Ethernet, CAN, CAN-FD, USB) & 2.95 \\
\hline 8 & Use of the Autonomous Vehicle toolbox in MATLAB / Simulink & 2.75 \\
\hline 9 & Self-driving vehicle ethics and legal issues & 2.71 \\
\hline 10 & Project and personnel management & 2.66 \\
\hline 11 & Functional safety & 2.62 \\
\hline 12 & Other & 2.18 \\
\hline
\end{tabular}


graduate and undergraduate students had a similar level of interest in independent study, which is equally applicable to both groups.

Finally, students were asked about their level of interest in the self-driving car industry, and their preparation to work in that industry. They rated their preparation and interest on a five-point Likert scale, with 5 denoting "strongly agree" and 1 representing "strongly disagree". Overall, students slightly disagreed with the statement that "The existing coursework at Kettering University has prepared me well to participate in the self-driving car industry", with an average rating of 2.81, and the distribution of rankings given in Figure 5.

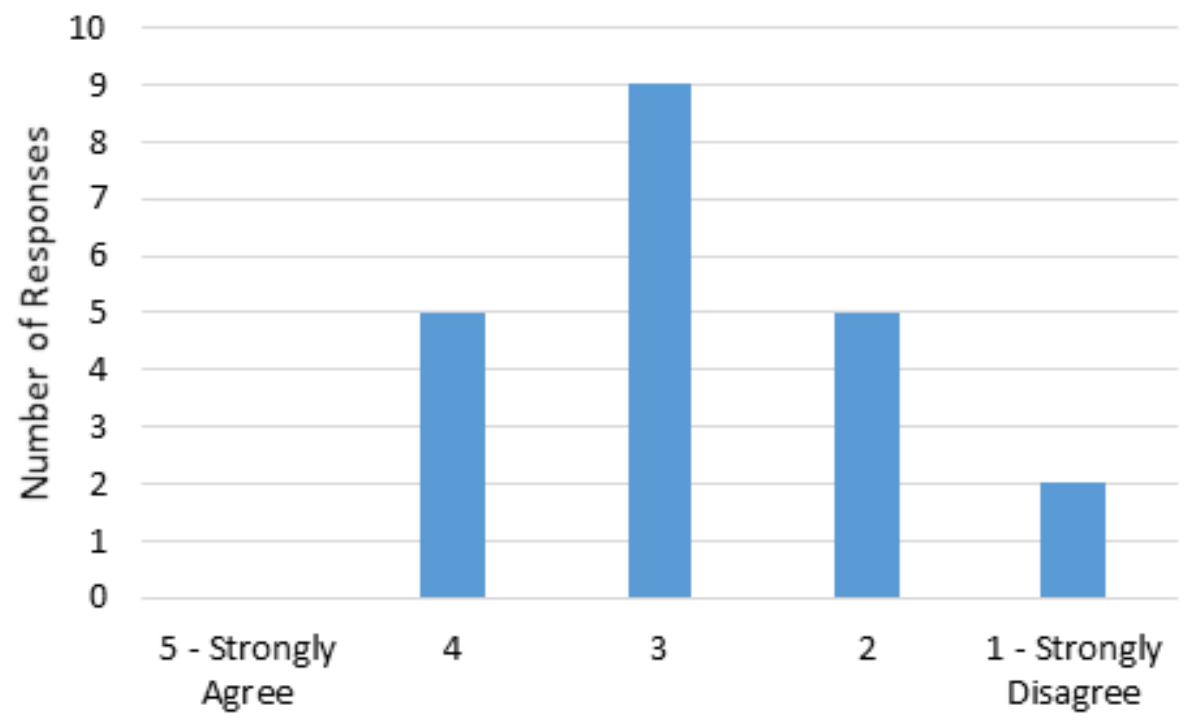

Figure 5: Preparation Provided by Coursework

Students did agree slightly with the statement that "Participation in the AutoDrive Challenge at Kettering University has prepared me well to work in the self-driving car industry", with an average rating of 3.62; the full distribution of rankings is given in Figure 6. These students are, overall, quite interested in working in this industry. When asked to indicate their agreement with the statement that "I have a strong interest in working in the self-driving car industry", the average ranking was 3.81, showing that the students on the AutoDrive team want the preparation they need to participate in this industry. The full distribution of rankings is given in Figure 7. These results indicate that, while competitions such as AutoDrive present a substantial educational opportunity to students, and that they value this opportunity, typical engineering and computer science courses are not seen as directly applicable to the industry. 


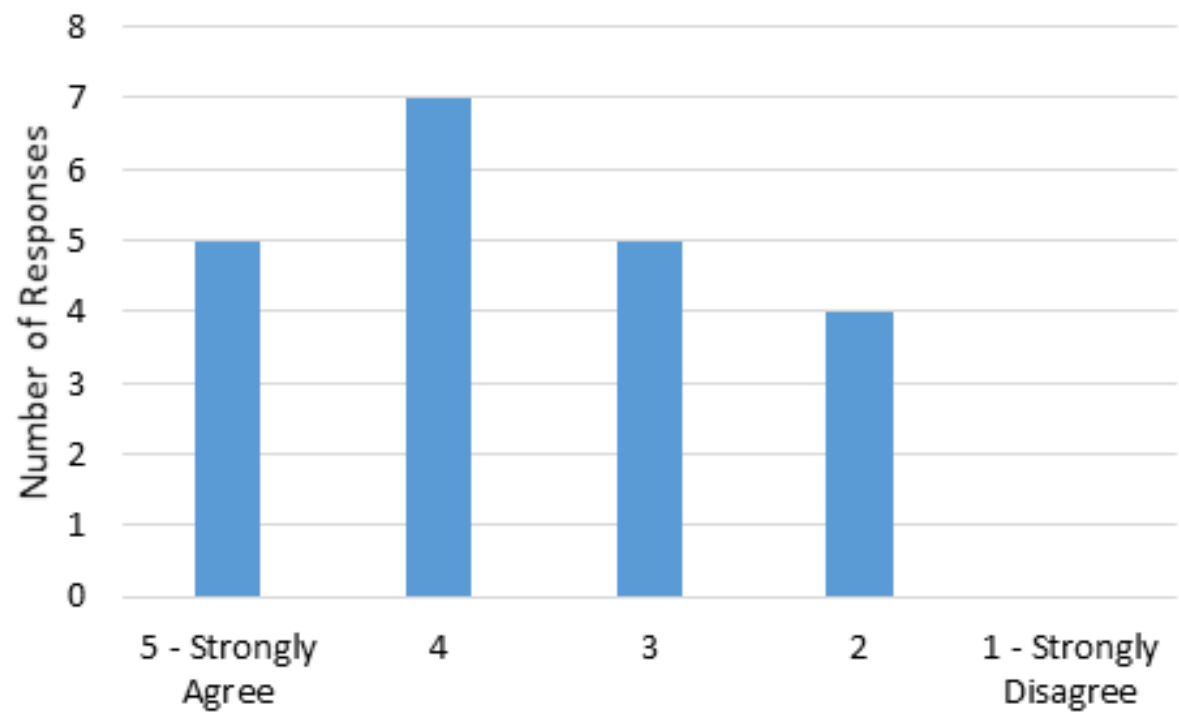

Figure 6: Preparation Provided by AutoDrive Participation

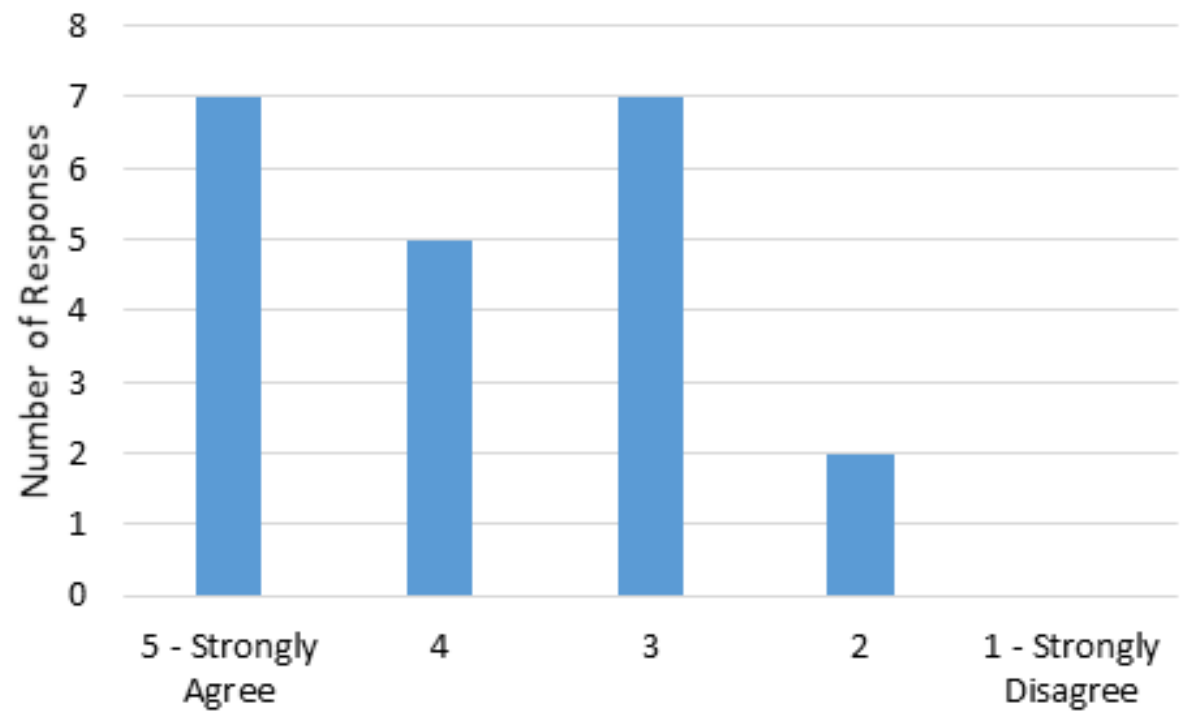

Figure 7: Interest Level in Self-Driving Car Industry

\section{Current Initiatives Within AutoDrive Addressing Education and Training Needs}

Education and training for autonomous vehicles at institutions around the world is in its early stages. There are a few isolated programs, offerings, and courses offered by various entities ranging from universities, training institutions, vendor companies, or industrial societies such as SAE. Although specific in its objectives, autonomous vehicle design draws from a wide variety of interdisciplinary fields, which makes it challenging to design appropriate education and training programs. What should be emphasized? Hardware, software, programming languages, artificial intelligence, car platforms, tools, software development frameworks, computing platforms, architectures, or other topics? The nature of some traditional - but content related - courses is 
changing due to rapid advances in the field. For example, the emergence of powerful frameworks for Deep Learning Neural Networks (DNN) such as Keras, Caffe, TensorFlow, Torch, Theano, and others requires less and less proficiency in programming languages, as the development tasks involve more configuration than programming. Another example is the emergence of powerful computing platforms such as CUDA, the related Open Computing Language ("OpenCL"), Field Programmable Gate Arrays ("FPGA"s), and hardware accelerators targeted to meet the stringent requirements of autonomous driving. All of this makes it challenging to design and offer compelling educational and training programs on autonomous driving. It is also difficult to balance the breadth and depth of foundational courses such as programming languages, image processing, computer vision, artificial intelligence, sensors, programming, and embedded systems.

Although all AutoDrive participating schools have been given a common set of hardware, software, and tools from GM and the main sponsors, each school has some freedom to use other solutions if so desired. This makes the task of education and training even harder because of the large number of options and possibilities open to the Teams. Given the short time frame of each competition (each spring of three consecutive years), in the case of Kettering University, students were forced to begin design and implementation work even if adequate education and training were not available or completed on time. Some of the topics for training are generic; for example, functional safety and systems engineering, while other topics are specific to the nature of the solution chosen, such as "end-to-end" versus model-based perception tasks. If the decision is end-to-end, then a great deal of education and training on neural networks and deep learning is necessary, together with schooling in powerful computing platforms such that the algorithms can run properly in real-time.

As the main organizer of AutoDrive, SAE provides technical assistance to all schools involved in the competition, primarily in the area of training. To accomplish this, SAE has organized specific training courses, seminars, workshops, and webinars, sometimes by itself and sometimes in cooperation with other competition sponsors. Several specific workshops have been organized by SAE at their headquarters in Pennsylvania, U.S.A. on important aspects of the competition. In addition, SAE has made available a few of their seminars to a limited number of students and faculty from each institution. One example is the seminar entitled Introduction to Highly Automated Vehicles [26], which was attended by one graduate student and one faculty member from Kettering University. SAE also offers a wide variety of courses in the automotive field, but few are relevant to autonomous vehicles; these courses include the following: Introduction to Radar for Automotive Applications, Wireless Communications in the Autonomous Connected Vehicle, and Cybersecurity: An Introduction for the Automotive Sector.

Sponsors also make available specific training on their hardware, software, or tools sometimes on their own, sometimes in cooperation with SAE. For example, Mathworks offers two-day training at SAE headquarters in Pennsylvania to a limited number of students and faculty participating in AutoDrive. Such training provides hands-on experience with developing and verifying automated driving perception algorithms, designing algorithms for Robot Operating System (ROS) enabled simulators and hardware, and appropriate MATLAB and toolbox functionality. Intel is another sponsor that has made available a number of webinars and workshops for AutoDrive participants on a wide variety of topics that include Open Source Computer Vision ("OpenCV”), OpenCL, 
Crystal Rugged Server, Arria 10 FPGA development kit, Accelerating OpenCV with Intel OpenCL for FPGAs, and Deep Learning Accelerator for Intel FPGA. Although there are other sponsors that are also vendors including GM, these have not yet provided specific education or training materials beyond product or service information and specifications.

Kettering University does not currently have academic or training programs specific to autonomous or connected vehicles. However, the school has developed a few courses to support AutoDrive, and it is in the process of reviewing its current programs in efforts to align them with this competition. Currently, planning is underway to develop concentrations and certificates in various areas that include autonomous mobility, connected mobility, and electrical mobility while focusing on autonomous driving, wireless vehicle connectivity, and electrical vehicles. Some school-wide generic courses on programming, control systems, and computer networking have been updated to include examples, laboratory experiments, and applications involving autonomous vehicles.

Along these lines, some courses have been redesigned or refocused while some new courses are being developed. The department of Electrical \& Computer Engineering ("ECE") has revised several of its courses to include examples and applications related to autonomous vehicles. In particular, a prior course on "Haptics" has been re-designed into a new course called Virtual Reality Systems: Modeling \& Control, to support simulation of autonomous vehicles in a virtual reality environment. This course provides the required theoretical and practical background for design and development of multi-modal Virtual Reality ("VR") systems. Particularly, the main focus is on VR-based human-in-the-loop systems that enable users to interact and/or manipulate virtual objects in simulated environments. This course aims to cover basics of these systems through lectures, homework, lab assignments, a term project, and readings on current related topics. Through lab assignments, students acquire hands-on skills to create a multi-modal virtual environment.

In addition, the ECE department has developed three new courses on computer vision, deep learning, and wireless communications and networking, with applications to autonomous vehicles. Important topics in these courses include: key technologies in autonomous driving, sensors and sensor architectures for autonomous driving, image representation, sampling and quantization, color systems (such as RGB, YCbCr, and HSL), and pixels and basic image filtering methods. Also highlighted are feature generation strategies, including edge detection methods, identification of interesting points, feature tracking and optical flow, structure from motion, image and region categorization, line detection, object detection, and tracking. Soft computing approaches are highlighted such as decision trees, nearest neighborhood method, clustering, K-means, neural networks (including multi-layer perceptrons), support vector machines, Adaboost, and deep neural networks. Additional topics include computer platforms, wireless communication and protocols, Dedicated Short Range Communication ("DSRC"), IEEE 802.11p and wave protocols, safety messages, and Vehicle-to-Vehicle ("V2V") and Vehicle-to-Infrastructure ("V2I") communications.

\section{Current Trends in Education and Training for Autonomous Vehicles}

Education and training in the development of autonomous vehicles plays an important role because many major automotive companies are planning to release autonomous vehicles by 2020 
and replace human driven cars by machines in the future [27]. Additionally, there is cutting-edge competition among companies to develop and train autonomous vehicles that can sense the environment, plan a path to a destination, and make correct and better decisions compared to human beings in the case of an emergency. Thus, autonomous vehicles are the future; to remain competitive in the automotive field, engineers will have to cultivate their knowledge of autonomous mobility.

There are several ways of learning the fundamental principles and systems running in the background of an autonomous vehicle, such as courses at universities, online paid and free courses, project-based learning offered at many universities as capstone design projects, seminars, workshops, conferences, and journals on autonomous vehicles. Education on autonomous vehicles is provided as courses in some universities in the form of conventional classes or independent studies. Massachusetts Institute of Technology provides a free course on Deep Learning for Self-Driving Cars [28], covering topics including self-driving vehicles, deep learning, reinforcement learning, computer vision, and human sensing. This course is available in two forms: in-person lecture and online lecture. University of Michigan, Ann Arbor also provides a four-day training program on Connected and Automated Vehicles (CAV) [29] that focuses on important topics such as safety standards and challenges faced by industry. Kettering University has also begun several courses on autonomous vehicles, to educate students on the latest developments in the field of autonomous vehicles, focusing especially on simulation on top of ROS. In Europe, a two-year Master degree course in autonomous vehicles is being taught by EIT Digital Master School with its centers in Hungary, Sweden, Finland, Italy, Germany, and France.

Teaching professionals can use open source software development platforms and libraries to design courses for autonomous vehicles; further developments can again be posted on these platforms for the use of other researchers. In this method, potential students and trainees can study algorithms and codes developed for autonomous vehicles using open source software development platforms. Several researchers publish their algorithms and codes, accessible to other researches, educators, teaching professionals, and students, for free on these platforms. GitHub [30] and Bitbucket [31] have been used to store these open source software development tools and algorithms. This process enables other researchers to develop their algorithms on top of already developed algorithms and thus advance the field of autonomous vehicles. There are many open source libraries available that can be used with different programming languages. These libraries provide a wide range of functions that can be used directly by researchers to hasten the development of autonomous vehicles.

A convenient method for gaining knowledge of autonomous vehicles is by taking online courses and tutorials. There are many companies that provide online services for learning using video lectures, and some of the online courses also provide a platform to practice and gain hands-on skills. For instance, one course available online on autonomous vehicles is Self-Driving Cars [32] by Udacity. Several courses are also provided by Coursera on Artificial Intelligence, Deep Learning, Conventional Neural Networks, and Machine Learning [33]. These courses are effective in providing knowledge for the development of algorithms for autonomous vehicles. Robot Ignite Academy provides a series of online courses for working with ROS, which is the operating system used in many autonomous vehicles. ROS Basics, ROS Navigation, ROS Perception, and ROS Autonomous Vehicles [34] are the courses provided, which are useful for 
working with ROS on autonomous vehicles and their simulation. All of these online courses and tutorials are good examples that can inspire educators in the field to develop more effective course materials.

Another method used by many universities is project-based learning. Some universities have a capstone design project that involves converting a radio-control car into an autonomous vehicle, which provides hands on experience to their students. Although the complexity of a real on-road autonomous vehicle is much greater than a small scale radio-control car, students learn basic concepts related to how a car can be made to think on its own and let it reach its destination autonomously. A current trend of this type involves developing a Nvidia Jetson based small-scale, self-driving car. These cars are created by students of several universities including Kettering University, Massachusetts Institute of Technology [35], University of Pennsylvania [36], and North Carolina A \& T State University [37]. The cars can be used to test basic algorithms to be applied to real world on-road autonomous vehicles. A further way of updating knowledge to the current level is by attending conferences and by reading conference proceedings and journal articles. For examples, IEEE organizes conferences like the Intelligent Vehicles Symposium [38], and International Conference on Intelligent Transportation Systems [39]. SAE organizes the Automated Driving Symposium [40].

Once a basic level of education about autonomous vehicles is gained, the next step is to enhance that knowledge and give something back to society in the form of research. Today, many universities have commenced their research on autonomous driving. Carnegie Mellon University in Pittsburgh, U.S.A. is considered by many as the birthplace of autonomous vehicles. This university has been working on autonomous vehicles for around 30 years, and ALVINN developed in 1989 - was one of the first autonomous vehicles. Stanford University has an autonomous driving research center called CARS, with connections to Google's self-driving vehicles project. The main areas of focus at Stanford are Artificial Intelligence, Machine Learning, and Deep Learning. While Massachusetts Institute of Technology focuses on sensor development and software for autonomous vehicles. MIT Media Lab has also collaborated with Toyota to perform research on secure data connection between vehicles.

Students from several universities have also collaborated to build autonomous vehicles, including the Princeton Autonomous Vehicle Engineering team ("PAVE"). This group has developed autonomous vehicles for the DARPA ("Defense Advanced Research Projects Agency") Grand Challenge and Intelligent Ground Vehicle Challenge [41]. Oxford University has the Oxford Robotics Institute with the stated mission as follows: "We are not condemned to a future of congestion, accidents and time wasting. We will eventually have cars that can drive themselves, interacting safely with other road users and using roads efficiently, thus freeing up our precious time." [42] Oxbotica is their spin-off company to develop driverless car technologies focusing on autonomous vehicles software.

Virtual reality simulators have also been used to create new knowledge about automated driving and examine new ideas related to the field. For example, a driving simulator has been developed at University of Warwick to test the real-world robustness and usability of smart, connected and autonomous vehicle technology [43]. Other universities including University of Southampton [44], Cambridge University [45], University of Leeds [46], and Technical University of Munich [47] have also conducted research in the field. Tsinghua University in 
China has expertise in the field of combining automated driving with electric powertrains. In 2016, the Intelligent Mobility research center opened in collaboration with Nissan, focusing on battery safety, autonomous driving and future traffic systems [48]. Seoul National University in Korea developed an autonomous car named SNUver at its Intelligent Vehicle Research Center. The car was equipped with 64 LIDAR sensors and it was tested on the busy roads of Korea [49].

All the methods of education and training have their own advantages and disadvantages. Online courses have the benefits of remote access and time flexibility, but they lack the human interaction that can be received in conventional classes. Online courses are also usually economical as compared to mainstream university courses. In the case of project-based learning, substantial resources, including space, technology and guidance, are required to execute a project. Open source software, libraries and repositories can be considered as a great means for research, as researchers may become acquainted with abundant data and many of the resources required at no cost. Ultimately, education and training play an important role in training experts who are required for advancing research and innovation in the development of autonomous vehicles. Education in automated driving can help graduate, undergraduate and high school students to develop their interest in the field of autonomous vehicles, which is the future of mobility.

\section{Discussion}

Although challenging, the design, implementation, and testing of a self-driving vehicle at SAE Level 4 in the context of a competition such as AutoDrive brings a host of benefits and value. In particular, project-based learning requires a great deal of resources to be truly successful. The approach taken by this competition is to leverage the work of students, faculty, and university administration. This is complemented by a host of resources, including a vehicle platform from GM, and other sensors, tools, and training, from SAE and other competition sponsors. The result is a high performing team backed up by the university, SAE, GM, and a host of competition sponsors.

AutoDrive brings many benefits to students, faculty, the university, sponsors, and the entire autonomous vehicle industry. Students develop valuable skills and experience in addition to taking courses and participating in training that is targeted on autonomous vehicles. Faculty get the opportunity to guide, advise, and consult with groups of students with real problems to solve, while directing a concerted effort to find solutions to difficult issues. The university views this as an opportunity to revisit its curriculum and create programs, certificates, and courses to address the education and training needs of the future workforce in autonomous vehicles. For the sponsors, AutoDrive is an inexpensive way to educate and train a workforce much needed in the industry. The entire autonomous vehicle industry benefits, because the competition will deliver results at a much lower cost than traditional means; for example, through funding start-ups. Additional benefits include a greater degree of collaboration between Kettering University and competition sponsors, and the identification and development of complementary research and activities in parallel to AutoDrive, to further the state of the art of self-driving vehicles. The competition also provides materials for case studies, and projects, that can be used in a variety of academic and training courses.

Some of the skills learned by the students include working as a team, practicing self-learning, learning about functional and system safety and system engineering concepts, using a variety of 
tools, implementing simulators, performing testing, and applying project management methods. Additional skills include learning to analyze, design, and evaluate the components of an autonomous vehicle including system integration. Other skills involve getting familiar with the technology behind autonomous vehicles, particularly perception systems, sensor fusion, control systems, by-wire systems, vehicle communications, artificial intelligence, and diagnostics. Together, these skills enable an increased level of knowledge and experience in designing and implementing an actual self-driving car. Thus far, the main outcomes of the AutoDrive Challenge at Kettering University are itemized as follows.

- Manufacture of a self-driving vehicle capable of successfully competing with the other Teams for the first year of the competition.

- Design and documentation of a complete system that endows a traditional vehicle with driver-less capabilities.

- Implementation of an infrastructure for educating and training students to design self-driving vehicles.

- Application of a process for faculty to act as advisors, coaches, and consultants to students involved in the competition.

- Creation of a set of educational and training programs, materials, and courses for educating and training students to design self-driving vehicles.

- Generation of an interdisciplinary community of students and faculty who would not have had the opportunity to work together otherwise.

Given the benefits of the competition, AutoDrive adds value in the context of the autonomous vehicle industry, as it address a need to prepare a well trained workforce in a university environment and produce real-world outcomes that meet stringent deadlines at extremely low cost with tangible benefits. An alternative way of producing these outcomes would be to form start-ups, which implies considerable costs to the industry. Our next steps consist of continuing to learn from the experiences afforded to our students, university, and faculty through competition, and incorporate these lessons learned in future endeavors. The steps include involving more students and stakeholders, improving educational and training initiatives at Kettering University, defining specific autonomous vehicles research projects that are separate from AutoDrive, and taking advantage of resources and opportunities effected through the process of participating in the competition. These resources and opportunities include an additional (self-funded) Chevrolet Bolt platform for research purposes, the General Motors Foundation Mobility Research Center, an on-campus proving ground, and donations from competition sponsors and Kettering University industrial partners.

\section{Summary and Conclusions}

AutoDrive is the latest Collegiate Design Series managed jointly by SAE and GM. Kettering University is one of eight schools participating in a three year competition to design and demonstrate a driver-less vehicle capable of navigating an urban environment at SAE Level 4. The driver-less vehicle will be entirely designed and developed by students, with faculty acting as coaches, advisors, and consultants in various capacities. Competition organizers and sponsors 
will fulfill important roles, including equipment donations and training. Currently, the competitor schools are preparing to participate in the second competition during May 2019 at the Mcity test facility in Ann Arbor, Michigan. AutoDrive is a great way to design an autonomous vehicle by leveraging the efforts of students, faculty, and administration of selected universities. It is also a fine course of action for educating and training students interested in working in the autonomous vehicles field. There are multiple benefits of the competition to all stakeholders. In summary, it adds great value to the autonomous vehicle industry.

Given that the AutoDrive Challenge only affects a small number of universities, how can the model, experience, and benefits be useful to other institutions? In the following we suggest some ways. Other universities might want to use a similar model for learning about education needs to apply to ongoing or future competitions, either on a small or large scale. The education and training needs identified here provide a means for other universities to prepare their students for working in the autonomous vehicle industry. The identified educational benefits can be used by faculty at other universities to persuade their institution and industrial stakeholders to be supportive of student competitions. Such competitions represent a significant value in undergraduate and graduate education; they should be viewed as a meaningful learning experience rather than just a "student club".

\section{Acknowledgment}

The authors would like to thank SAE, GM, and all of our sponsors, donors, and partners.

\section{References}

[1] S. Thrun et al., "Stanley: The robot that won the DARPA grand challenge," Journal of Field Robotics, vol. 23, no. 9, pp. $661-692,2006$.

[2] C. Urmson et al., "Autonomous driving in urban environments: Boss and the urban challenge," Springer Tracts in Advanced Robotics, vol. 56, pp. 1 - 59, 2009.

[3] P. C. Wankat, "Undergraduate student competitions," Journal of Engineering Education, vol. 94, no. 3, pp. 343 - 347, 2005.

[4] D. M. Reimer, A. Ali, and S. R. Abro, "Relationship between student competitive activities and the entrepreneurial mindset," in ASEE Annual Conference and Exposition, Conference Proceedings, Vancouver, BC, Canada, 2011.

[5] C. Carroll, "Competition based learning in the classroom," in ASEE Annual Conference and Exposition, Conference Proceedings, Atlanta, GA, U.S.A., 2013.

[6] R. Beach et al., "Robotics innovations competition and conference (RICC): Building community between academia and industry through a university-level student competition," in ASEE Annual Conference and Exposition, Conference Proceedings, Louisville, KY, U.S.A., 2010.

[7] P. Schuster, A. Davol, and J. Mello, "Student competitions - The benefits and challenges," in ASEE Annual Conference and Exposition, Conference Proceedings, Chicago, IL, U.S.A., 2006. 
[8] P. W. Wickenden and R. K. Stobart, "Integrating Formula SAE with the engineering curriculum," in SAE Technical Paper No. 2005-01-1796.

[9] K. G. Wolfinbarger and R. L. Shehab, "What behaviors and characteristics do engineering student competition team members associate with leadership?" in ASEE Annual Conference and Exposition, Conference Proceedings, Seattle, WA, U.S.A., 2015.

[10] F. G. Gonzalez, "Systems engineering education through participation in engineering competitions," in ASEE Annual Conference and Exposition, Conference Proceedings, San Antonio, TX, U.S.A., 2012.

[11] SAE International, Ground Vehicle Standard J3016: Taxonomy and Definitions for Terms Related to Driving Automation Systems for On-Road Motor Vehicles, 2016.

[12] I. Dabipi and J. Arumala, "Enhancing engineering education through real-life projects," in ASEE Annual Conference and Exposition, Conference Proceedings, Honolulu, HI, U.S.A., 2007.

[13] J. Bringardner, "Developing a vertically integrated project course to connect undergraduates to graduate research projects on smart cities transportation technology," in ASEE Annual Conference and Exposition, Conference Proceedings, Columbus, OH, U.S.A., 2017.

[14] S. C. Dunning, C. Aldrich, and M. Webber, "The Holden elementary school autonomous vehicle (HESAV)," in ASEE Annual Conference and Exposition, Conference Proceedings, Nashville, TN, U.S.A., 2003.

[15] M. E. Holden, "Low-cost autonomous vehicles using just GPS," in ASEE Annual Conference and Exposition, Conference Proceedings, Salt Lake City, UT, U.S.A., 2004.

[16] T. B. Stout and S. C. Hsiung, "Collaborated process with a wireless autonomous vehicle at its center," in ASEE Annual Conference and Exposition, Conference Proceedings, Indianapolis, IN, U.S.A., 2014.

[17] N. K. Lape et al., "Autonomous vehicles: A hands-on interdisciplinary freshman course," in ASEE Annual Conference and Exposition, Conference Proceedings, Vancouver, BC, Canada, 2011.

[18] P. Giolma, F. Aminian, and D. Ibaroudene, "An autonomous robot - The ideal design project?" in ASEE Annual Conference and Exposition, Conference Proceedings, Charlotte, NC, U.S.A., 1999.

[19] F. Rios-Gutierrez and R. Alba-Flores, "Mobile robots capstone design course," in ASEE Annual Conference and Exposition, Conference Proceedings, Chicago, IL, U.S.A., 2006.

[20] R. Y. Chiou, Y. Ertekin, and T.-L. B. Tseng, "MAKER: Autonomous solar-powered vehicle as a learning tool in robotics and green energy," in ASEE Annual Conference and Exposition, Conference Proceedings, New Orleans, LA, U.S.A., 2016.

[21] B. H. Riley, C. Schumann, and J. Petersen, "IR sensing integrated with a single board computer for development and demonstration of autonomous vehicle following," in ASEE Annual Conference and Exposition, Conference Proceedings, Columbus, OH, U.S.A., 2017. 
[22] H. Cheng, Autonomous Intelligent Vehicles: Theory, Algorithms, and Implementation. London: Springer, 2011.

[23] F. Y. Wang et al., "IVS O5: New developments and research trends for intelligent vehicles," IEEE Intelligent Systems, vol. 20, no. 4, pp. 10 - 14, 2005.

[24] H. Cheng et al., "Interactive road situation analysis for driver assistance and safety warning systems: Framework and algorithms," IEEE Transactions on Intelligent Transportation Systems, vol. 8, no. 1, pp. 157 - 166, 2007.

[25] A. Geiger et al., "Vision meets robotics: The KITTI dataset," International Journal of Robotics Research, vol. 32, no. 11, pp. 1231 - 1237, 2013.

[26] [Online], Available: http://training.sae.org/seminars/c1603/, Accessed Mar. 17, 2019.

[27] J. B. Greenblatt and S. Shaheen, "Automated vehicles, on-demand mobility, and environmental impacts," Current Sustainable/Renewable Energy Reports, vol. 2, no. 3, pp. 74 - 81, 2015, [Online], Available: https://doi.org/10.1007/s40518-015-0038-5, Accessed Mar. 17, 2019.

[28] [Online], Available: https://selfdrivingcars.mit.edu/, Accessed Mar. 17, 2019.

[29] [Online], Available: http://isd.engin.umich.edu/professional-programs/connected-andautomated-vehicles/index.htm, Accessed Mar. 17, 2019.

[30] [Online], Available: https://github.com/, Accessed Mar. 17, 2019.

[31] [Online], Available: https://bitbucket.org/, Accessed Mar. 17, 2019.

[32] [Online], Available:

https://www.udacity.com/course/self-driving-car-engineer-nanodegree--nd013, Accessed Mar. 17, 2019.

[33] [Online], Available:

https://www.coursera.org/courses?languages=en\&query=self-driving+car, Accessed Mar. $17,2019$.

[34] [Online], Available: http://www.theconstructsim.com/construct-learn-develop-robots-usingros/robotigniteacademy_learnros/, Accessed Mar. 17, 2019.

[35] [Online], Available:

http://www.jetsonhacks.com/2015/10/06/mit-racecar-walkthrough-nvidia-jetson-tk1/, Accessed Mar. 17, 2019.

[36] [Online], Available: http://www.jetsonhacks.com/2016/06/13/jetson-based-autonomousrace-car-university-pennsylvania/, Accessed Mar. 17, 2019.

[37] [Online], Available:

http://www.jetsonhacks.com/2017/03/02/daniel-tobias-car-cherry-autonomous-racecar/, Accessed Mar. 17, 2019.

[38] [Online], Available: https://www.ieee-itss.org/iv, Accessed Mar. 17, 2019. 
[39] [Online], Available: https://www.ieee-itsc2018.org/, Accessed Mar. 17, 2019.

[40] [Online], Available: https://www.sae.org/attend/, Accessed Mar. 17, 2019.

[41] [Online], Available: https://pave.princeton.edu/, Accessed Mar. 17, 2019.

[42] [Online], Available: http://ori.ox.ac.uk/about-us/, Accessed Mar. 17, 2019.

[43] [Online], Available: https://warwick.ac.uk/fac/sci/wmg/mediacentre/wmgnews/?newsItem= 094d43455534bb5d0155a24263d173c2, Accessed Mar. 17, 2019.

[44] [Online], Available:

https://www.southampton.ac.uk/news/2015/10/driverless-cars-epsrc-funding.page, Accessed Mar. 17, 2019.

[45] [Online], Available: http://www.cauv.co.uk/, Accessed Mar. 17, 2019.

[46] [Online], Available:

http://www.leeds.ac.uk/site/custom_scripts/spotlight/driverless-cars.php, Accessed Mar. 17, 2019.

[47] [Online], Available:

https://www.tum.de/en/about-tum/news/press-releases/detail/article/33216/, Accessed Mar. $17,2019$.

[48] [Online], Available: http://nissannews.com/en-US/nissan/usa/releases/nissan-joint-researchcenter-for-intelligent-mobility-at-tsinghua-university-opens-in-beijing? mode=print, Accessed Mar. 17, 2019.

[49] [Online], Available: http://pulsenews.co.kr/view.php?year=2017\&no=216397, Accessed Mar. 17, 2019. 